\title{
Possible Disadvantages of Gonadal Suppression Therapy
}

\author{
Katsuhiko Tachibana \\ Department of Endocrinology and Metabolism, Kanagawa Children's Medical Center, Yokohama, Japan
}

\begin{abstract}
Gonadal suppression therapy is now used experimentally to improve the final height of children who enter puberty at the normal age but with short stature. For this purpose a long-acting analog of LH-releasing hormone (LHRHa) is usually used. To improve the final height, gonadal suppression must be continued for at least several years, and artificial delayed puberty may cause psychosocial problems. The accumulation of bone mineral is reported to be most active during puberty and it is related to the increased secretion of sex steroids. Suppression of sex steroid secretion at the normal pubertal age may result in poor bone mineral accumulation at this critical period and may become a risk factor for osteoporosis later in life. Gonadotropins are known to be secreted in a pulsatile fashion even at prepubertal age. LHRHa abolishes gonadotropin secretion completely and may deteriorate pubertal development after completion of the therapy. There are reports of IgE antibody production and anaphylaxis caused by LHRHa treatment. If an antibody that cross-reacts with native LHRH is generated, it can be a cause of hypogonadism. We should take into account these possible disadvantages when giving gonadal suppression therapy to patients with basically normal puberty.
\end{abstract}

Key words: gonadal suppression, LHRH analog, side effect, final height, precocious puberty

Gonadal suppression therapy is basically a treatment for precocious puberty. The purposes of this therapy are, first, to resolve psychosocial problems arising from the precocious second sexual characteristics by suppressing their progression and, second, to improve final height otherwise expected to be stunted by the precocious bone maturation.

Sexual precocity is defined as in Table 1 . In brief, if a patient meets 2 or 3 of these criteria, he can be diagnosed as a case of precocious puberty. The diagnosis of precocious puberty does not necessarily mean the patient must be treat-

Correspondence: Dr. Katsuhiko Tachibana, Department of Endocrinology and Metabolism, Kanagawa Children's Medical Center, 2-138-4, Mutsukawa, Minami-ku, Yokohama 232, Japan ed. Even when secondary sexual characteristics appear early, if the patient has already attained a certain height and is not expected to be an extremely short adult, the gonadal suppression therapy is not necessary unless precocity causes psychosocial problems. On the other hand, even the appearance of second sexual characteristics is normal for chronological age if the height is still short; in other words, if the patient is precocious for his height age, the final height may be extremely short. Some researchers believe in taking such a situation as relative precocity and introducing gonadal suppression therapy to improve final height. Relative precocity is not unusual among the patients with growth hormone deficiency, IUGR and normal short stature.

In this paper I wish to mention the possible disadvantages of gonadal suppression therapy 
Table 1 Definition of sexual precocity

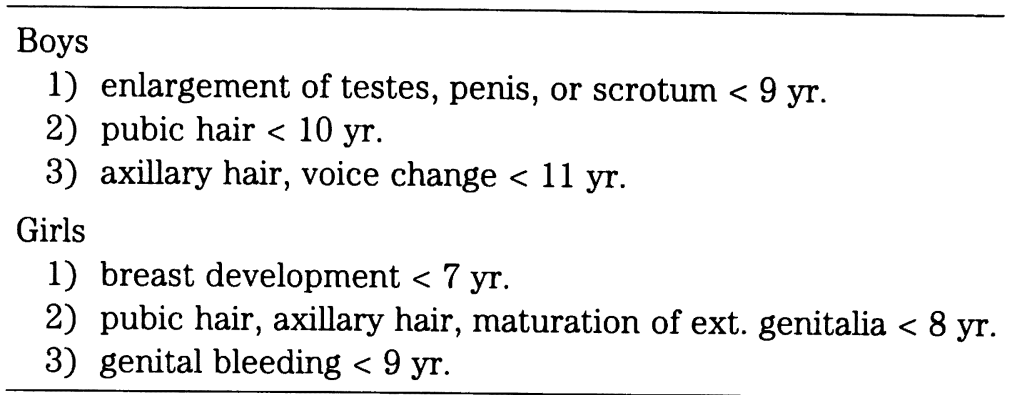

which we should bear in mind when introducing it with the aim of improving final height in (relative) precocious puberty.

\section{Effects}

As the gonadal suppression therapy aimed at improving final height, a long-acting analog of LH-releasing hormone (LHRHa) is usually used these days. LHRHa suppresses the gonadotropin secretion and decreases the secretion of sex steroids, and growth rate decreases to the prepubertal level when the patient has already begun the pubertal growth spurt. The tactic of gonadal suppression therapy is to prevent the bone maturation and to elongate the time before reaching final height although apparent growth velocity decreases, but, in some cases, growth velocity is suppressed to less than the prepubertal level. This is supposed to be because LHRHa suppresses GH secretion also (1). In such cases, combined therapy with GH may be effective (2), but GH is not approved to be used in such cases in Japan and treating basically normal children with GH and LHRHa raises an ethical issue. We should restrict the subjects to those who are supposed to become extremely short adults.

As a gonadal suppression therapy, cyproterone acetate has been used, and shortly after it was introduced there appeared many reports saying that cyproterone is effective in improving the final height of patients with precocious puberty. These were based on the fact that cyproterone improves the predicted adult height and suppresses the bone maturation (decreases the ratio of bone age increase to chronological age increase $(\triangle \mathrm{BA} / \Delta \mathrm{CA}))$, but these days cyproterone is reported to be ineffective in improving final height in precocious puberty based on the actual final height of treated patients. LHRHa is now reported to be effective in improving final height, but this is mainly based on the improvement of predicted adult height or a decreased $\Delta \mathrm{BA} / \Delta \mathrm{CA}$ ratio. Whether LHRHa is truly effective in improving final height or not is not still clear.

\section{Artificial Delayed Puberty}

To improve final height, gonadal suppression therapy must be continued for at least several years. In cases of relative precocious puberty, the subjects are not precocious for their chronological age, and gonadal suppression produces artificial delayed puberty, and this may cause psychosocial problems.

\section{Bone Mineral Accumulation}

Puberty is the time when bone mineral accumulation is higher than at any other time in life (3), and this is supposed to be due to the influence of increased sex steroid secretion. Poor bone mineral accumulation during puberty is reported to be one of the most important risk factors for osteoporosis later in life. After pubertal age, bone mineral accumulation becomes 
almost negligible even though sex steroid secretion continues (3). Artificially delayed puberty may deteriorate the normal bone mineral accumulation during this critical period. It is possible that, after the completion of treatment, this active bone mineral accumulation does not take place any more in the face of increased sex steroid secretion, since the critical age (bone age or chronological age) has already passed. Although we must await the results of further studies to know whether this is true or not, there is a report stating that patients with delayed puberty have low bone mineral density after maturation (4).

\section{Recovery of Gonadal Function}

During LHRHa treatment, the patient is in a state of hypogonadotropic hypogonadism. With long-standing hypogonadotropic hypogonadism, the gonads might become disuse atrophy. In an experimental animal, histological change was observed after long term treatment with LHRHa (5). In the case of boys, once enlarged testes shrink due to LHRHa treatment (6), it is possible that such testes become fibrotic and cannot enlarge again after the cessation of treatment, and spermatogenesis might be impaired despite normal testosterone production.

It is reported that pulsatile gonadotropin secretion is present even during the prepubertal period although the serum concentration is low. The importance of this pulsatile secretion is not yet known. During LHRHa treatment, gonadotropin secretion is abolished almost completely and the effect of this complete suppression on later development of gonadal function is not known.

There is a report of the production of IgE antibody to LHRHa (7). If an antibody that crossreacts with native $\mathrm{LHRH}$ and interferes with its activity is produced, it may result in hypogonadism.

It is now believed that the recovery of gonadal function after the cessation of LHRHa therapy is prompt in view of the experience of precocious puberty. But since permanent hypogonadism after LHRHa therapy cannot be excluded completely, we must be aware of this possibility especially when introducing the treatment for relative precocity aiming only to improve final height.

\section{Side Effects}

The report of $\operatorname{IgE}$ production has already been mentioned. Besides this, a case of anaphylaxis was reported (8). There are reports of a high incidence of hives or wheal formations (7, $9,10)$. An aseptic abscess at the injection site has also been reported (9).

Side effects of LHRHa are not reported frequently, but it is a relatively new drug and we should observe it carefully. For example, we used cyproterone acetate for gonadal suppression before LHRHa became available. Although we knew the possibility of adrenal suppression of cyproterone, an unexpected possible side effect has emerged recently: three cases of hepatocellular carcinoma (HCC) were reported among patients treated with cyproterone (11). The relation between HCC and cyproterone is still under the investigation. Such side effects may appear with LHRHa in future.

LHRHa stimulates the gonadotropin secretion transiently at the beginning of the treatment because of its nature. It is well known that temporal progression of second sexual characteristics and withdrawal bleeding are occasionally observed shortly after the initiation of LHRHa therapy. At the start of LHRHa therapy for girls, we repeat ultrasonography of the ovaries and have detected enlargement of ovarian follicles not infrequently. Figure 1 shows an extreme case. The patient is an 8-year-old girl and LHRHa nasal spray was introduced following a diagnosis of precocious puberty. Two weeks after the start of the treatment, a follicle in the right ovary was found to be enlarged on ultrasonography. At three weeks the cystic lesion enlarged further 


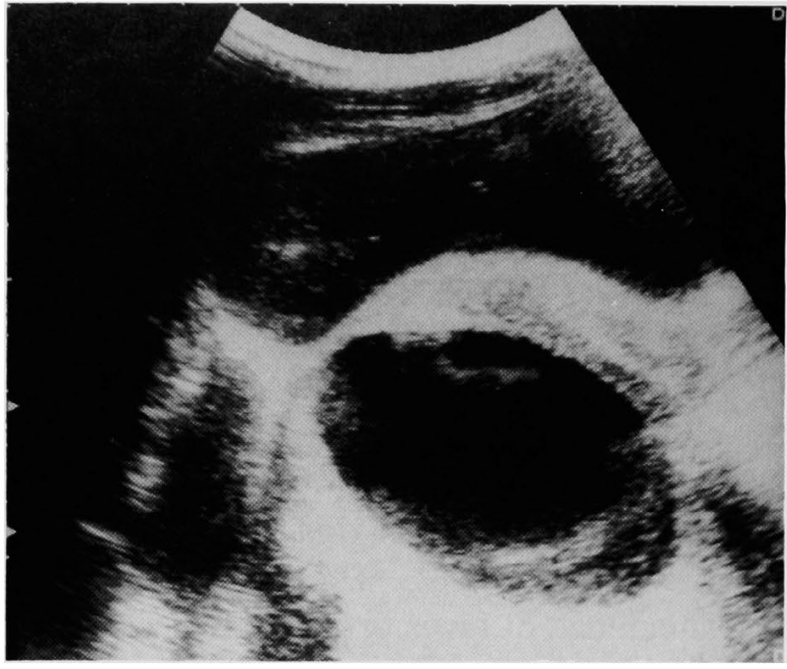

Fig. 1 The pelvic ultrasonography of an 8-year-old girl with precocious puberty at three weeks after the initiation of intranasal LHRH analog administration.

and its diameter was about $9 \mathrm{~cm}$. Fortunately the cyst shrank later without incident. In such cases, rupture of the cyst may occur.

The disadvantages I mentioned here are merely possibilities and not necessary encountered. Any medication has its disadvantages, and doctors introduce it when the expected therapeutic advantages are supposed to be greater than possible disadvantages. To improve final height is very important for patients of short stature and gonadal suppression therapy may be necessary in some patients. But we should always bear in mind the possible disadvantages of gonadal suppression therapy especially when introducing it only to improve final height and restrict it to patients who otherwise would be expected to become extremely short adults.

\section{References}

1. DiMartino-Nardi J, Wu R, Fishman K, Saenger P. The effect of long-acting analog of luteinizing hormone-releasing hormone on growth hormone secretory dynamics in children with precocious puberty. J Clin Endocrinol Metab 1991; 73: 902-6.

2. Tanaka T, Satoh M, Hibi I. Combined GH and LHRH analog treatment can increase pubertal growth in short children. Clin Pediatr Endocrinol 1997; 6 (suppl): 39-44.

3. Theintz G, Buchs B, Rizzoli R, Slosman D, Clavien $\mathrm{H}$, Sizonenko $\mathrm{PC}$, et al. Longitudinal monitoring of bone mass accumulation in healthy adolescents: Evidence for a marked reduction after 16 years of age at the levels of lumbar spine and femoral neck in female subjects. J Clin Endocrinol Metab 1992; 75: 1060-5.

4. Finkelstein JS, Neer RM, Biller BMK, Crawford JD, Klibanski A. Osteopenia in men with a history of delayed puberty. New Eng J Med 1992; 326: $600-4$.

5. MavGillvray MH, Treatment of idiopathic precocious puberty. New Eng J Med 1982; 306: 1109-10.

6. Rime JL, Zumsteg U, Blumberg A, et al. Longterm treatment of central precocious puberty with an intranasal LHRH analogue: control of pituitary function by urunary gonadotropins. Eur J Pediatr 1988; 147: 263-9.

7. Styne DM, Harris DA, Egli CA, Conte FA, Kaplan $\mathrm{SL}$, Rivier J, et al. Treatment of true precocious puberty with a potent luteinizing hormone-releasing factor agonist: Effect on growth, sexual maturation, pelvic sonography, and the hypothalamic-pituitary-gonadal axis. J Clin Endocrinol Metab 1985; 61: 142-51.

8. Letterie GS, Stevenson D, Shah A. Recurrent anaphylaxis to a depot form of GnRH analogue. Obstet Gynecol 1991; 78: 943-6.

9. Breyer P, Haider A, Pescovitz $\mathrm{OH}$. Gonadotropin-releasing hormone agonists in the treatment of girls with central precocious puberty. Clin Obstet Gynecol 1993; 36: 764-72.

10. Kaplan SL, Grumbach MM. True precocious puberty: treatment with GnRH agonists. In: Delemarre Van deWall HA, editors, Control of the onset of puberty. Amsterdam: Elsevier Science Publishers; 1989: 357-73.

11. Watanabe S, et al. Three cases of hepatocellular carcinoma among cyproterone users. Lancet 1994; 344: 1567. 